\title{
Treatments used for obsessive-compulsive disorder-An international perspective
}

\author{
Vlasios Brakoulias $^{1}$ (D) | Vladan Starcevic ${ }^{1}$ | Umberto Albert ${ }^{2,3}$ | Shyam Sundar Arumugham ${ }^{4}$ | \\ Brenda E. Bailey ${ }^{5}$ | Amparo Belloch ${ }^{6}$ | Tania Borda ${ }^{7,8}$ | Liliana Dell'Osso ${ }^{9}$ | Jason A. Elias ${ }^{10}$ | \\ Martha J. Falkenstein ${ }^{10}$ | Ygor A. Ferrao ${ }^{11}$ | Leonardo F. Fontenelle ${ }^{12,13,14}$ | Lena Jelinek ${ }^{15}$ | \\ Leto Kalogeraki ${ }^{16}$ | Brian Kay ${ }^{5}$ | Luana D. Laurito ${ }^{12,13,14}$ | Christine Lochner ${ }^{17}$ | \\ Giuseppe Maina ${ }^{3}$ | Donatella Marazziti ${ }^{9}$ (D) | Andrew Martin ${ }^{18}$ | Hisato Matsunaga ${ }^{19}$ | \\ Euripedes C. Miguel ${ }^{20}$ | Pedro Morgado ${ }^{21,22,23}$ | Irakis Mourikis ${ }^{16}$ | Massimo Pasquini ${ }^{24}$ | \\ Rodrigo Perez Rivera ${ }^{7}$ | Sriramya Potluri ${ }^{10}$ | Janardhan Y.C. Reddy ${ }^{4}$ Brian C. Riemann ${ }^{5}$ | \\ Maria Conceição do Rosario ${ }^{25}$ | Roseli G. Shavitt ${ }^{11}$ | Dan J. Stein ${ }^{17}$ | Kirupumani Viswasam ${ }^{1}$ | \\ Zhen Wang ${ }^{26,27}$ । Naomi A. Fineberg ${ }^{28}$
}

\footnotetext{
${ }^{1}$ Department of Psychiatry, Nepean Hospital, Sydney Medical School, The University of Sydney, Penrith, NSW, Australia

${ }^{2}$ Rita Levi Montalcini Department of Neuroscience, University of Turin, Turin, Italy

${ }^{3}$ Department of Biomedical and Neuromotor Sciences, Alma Mater Studiorum University of Bologna, Bologna, Italy

${ }^{4}$ Department of Psychiatry, National Institute of Mental Health and Neurosciences (NIMHANS), Bengaluru, India

${ }^{5}$ Department of Psychiatry, Rogers Memorial Hospital, Oconomowoc, Wisconsin

${ }^{6}$ Department of Personality Psychology, Research Unit for Obsessive-Compulsive and Related Disorders, I'TOC, Faculty of Psychology, Universidad de Valencia, Valencia, Spain

${ }^{7}$ Department of Psychiatry, Bio-Behavioral Institute BA, Buenos Aires, Argentina

${ }^{8}$ Department of Psychology, Argentinian Catholic University (UCA), Buenos Aires, Argentina

${ }^{9}$ Dipartimento di Medicina Clinica e Sperimentale, Section of Psychiatry, Dipartmento di Farmacia, University of Pisa, Pisa, Italy

${ }^{10}$ Department of Psychiatry, McLean Hospital/Harvard Medical School, Belmont, Massachusetts

${ }^{11}$ Department of Psychiatry, Federal University of Health Sciences of Porto Alegre, Porto Alegre, Brazil

${ }^{12}$ Department of Psychiatry and Legal Medicine, Federal University of Rio de Janeiro, Rio de Janeiro, Brazil

${ }^{13}$ Department of Psychiatry, D'Or Institute for Research and Education, Rio de Janeiro, Brazil

${ }^{14}$ Monash Institute of Cognitive and Clinical Neurosciences, Monash University, Clayton, Victoria

${ }^{15}$ Department of Psychology, Universitätsklinikum Hamburg-Eppendorf, Hamburg, Germany

${ }^{16}$ Behavioral Therapy Department/Outpatient Clinic for OCD and Related Disorders, Division of Psychiatry I, National and Kapodistrian University of Athens, Athens, Greece

${ }^{17}$ MRC Unit on Anxiety Disorders, Department of Psychiatry, University of Stellenbosch, Cape Town, South Africa

${ }^{18}$ NHMRC Clinical Trials Centre, The University of Sydney, Sydney, NSW, Australia

${ }^{19}$ Department of Neuropsychiatry, Hyogo College of Medicine, Nishinomiya, Japan

${ }^{20}$ Department and Institute of Psychiatry, University of São Paulo, São Paulo, Brazil

${ }^{21}$ Life and Health Sciences Research Institute (ICVS), School of Health Sciences, University of Minho, Braga, Portugal

${ }^{22}$ Department of Psychiatry, ICVS-3Bs-PT Government Associate Laboratory, Braga/Guimarães, Portugal

${ }^{23}$ Department of Psychiatry, Hospital de Braga, Braga, Portugal

${ }^{24}$ Department of Human Neurosciences, Sapienza University of Rome, Rome, Italy

${ }^{25}$ Department of Psychiatry, Federal University of São Paulo, São Paulo, Brazil

${ }^{26}$ Shanghai Mental Health Center, Shanghai Jiao Tong University School of Medicine, Shanghai, China

${ }^{27}$ Department of Psychiatry, Shanghai Key Laboratory of Psychotic Disorders, Shanghai, China

${ }^{28}$ Department of Psychiatry, Hertfordshire Partnership University NHS Foundation Trust and University of Hertfordshire, Hatfield, UK
} 


\section{Correspondence}

Dr. Vlasios Brakoulias, Department of Psychiatry, Nepean Hospital, Sydney Medical School, The University of Sydney, PO Box 63, Penrith, NSW 2751, Australia.

Email: vbrakoulias@bigpond.com; vlasios. brakoulias@health.nsw.gov.au

Funding information

Spanish MINECO, Grant/Award Number: PSI2013-44733-R; National Natural Science Foundation of China, Grant/Award Number: 81371340; Pfizer Neuroscience Grant; Nepean Medical Research Foundation Grant

\section{Abstract}

Objective: The objective of this study was to characterise international trends in the use of psychotropic medication, psychological therapies, and novel therapies used to treat obsessive-compulsive disorder (OCD).

Methods: Researchers in the field of OCD were invited to contribute summary statistics on the characteristics of their samples. Consistency of summary statistics across countries was evaluated.

Results: The study surveyed 19 expert centres from 15 countries (Argentina, Australia, Brazil, China, Germany, Greece, India, Italy, Japan, Mexico, Portugal, South Africa, Spain, the United Kingdom, and the United States) providing a total sample of 7,340 participants. Fluoxetine $(n=972 ; 13.2 \%)$ and fluvoxamine $(n=913 ; 12.4 \%)$ were the most commonly used selective serotonin reuptake inhibitor medications. Risperidone $(n=428 ; 7.3 \%)$ and aripiprazole $(n=415 ; 7.1 \%)$ were the most commonly used antipsychotic agents. Neurostimulation techniques such as transcranial magnetic stimulation, deep brain stimulation, gamma knife surgery, and psychosurgery were used in less than $1 \%$ of the sample. There was significant variation in the use and accessibility of exposure and response prevention for OCD.

Conclusions: The variation between countries in treatments used for OCD needs further evaluation. Exposure and response prevention is not used as frequently as guidelines suggest and appears difficult to access in most countries. Updated treatment guidelines are recommended.

\section{KEYWORDS}

antipsychotics, benzodiazepines, cross-cultural study, obsessive-compulsive disorder, pharmacotherapy, selective serotonin reuptake inhibitors

\section{1 | INTRODUCTION}

Obsessive-compulsive disorder (OCD) is characterised by repetitive and intrusive thoughts, urges, images or fears (obsessions), and repetitive behaviours or mental acts (compulsions). Common symptoms include fears of contamination and excessive hand washing, preoccupation with symmetry and ordering, intrusive and distressing unacceptable or taboo thoughts, and repetitive checking. These types of symptoms tend to be similar regardless of cultural background (Matsunaga et al., 2008). Throughout the world, OCD is thought to occur in 0.8 to $2 \%$ of the population (Ruscio, Stein, Chiu, \& Kessler, 2010). It is viewed as a relapsing remitting disorder (Eisen et al., 2013; Skoog \& Skoog, 1999), and patients with OCD often do not receive optimal treatment (Sorsdahl et al., 2013). Treatment guidelines (Baldwin et al., 2014; Bandelow et al., 2012; Marazziti \& Consoli, 2010; National Institute for Health and Clinical Excellence, 2005) recommend selective serotonin reuptake inhibitors (SSRIs) or exposure and response prevention (ERP) as first-line treatments. However, response to pharmacological treatment is frequently inadequate (Schruers, Koning, Luermans, Haack, \& Griez, 2005) and further limited by poor insight, medication nonadherence, and/or adverse effects.

Cross-cultural studies of OCD have been encouraged (Stein \& Rapoport, 1996), and research assessing international prescribing trends for OCD can inform us whether treatment preferences vary from one country to another. Building on the findings of a previous international survey (Brakoulias et al., 2016), this study thus had three aims: (a) to compare the frequencies of psychotropic agent use for OCD across a larger number of countries, with a more specific focus on the types of SSRIs and antipsychotics and other pharmacological agents used in each country, (b) to report on the use of novel therapeutic modalities, for example, transcranial magnetic stimulation (TMS), in OCD, and (c) to determine the frequency of use of ERP in $O C D$ and its accessibility. Based on existing literature, study hypotheses were as follows: (a) Use of pharmacological agents for OCD varies significantly between countries (Brakoulias et al., 2016; Van Ameringen et al., 2014), (b) SSRIs are the most commonly prescribed pharmacotherapeutic agents for OCD (Brakoulias et al., 2013; Brakoulias et al., 2016), and (c) ERP is used less frequently in countries where access to trained therapists is limited and costly.

\section{2 | METHODS}

The first author V. B. wrote to leading international OCD researchers, asking them to take part in the large international survey. These researchers worked predominantly in publicly funded specialised 


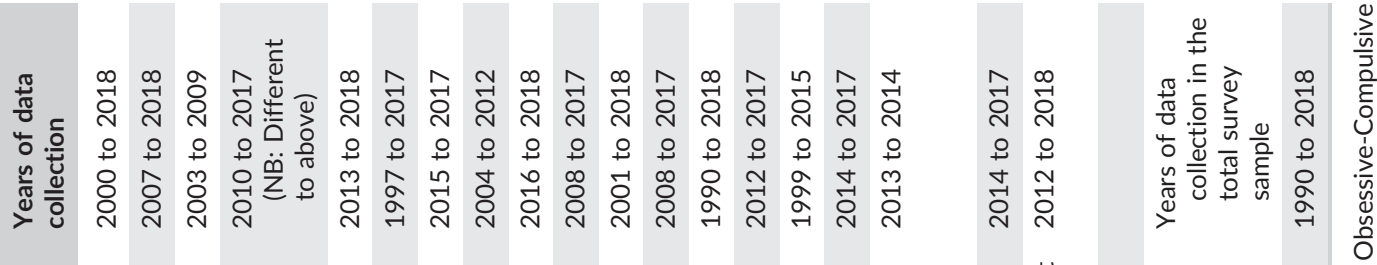

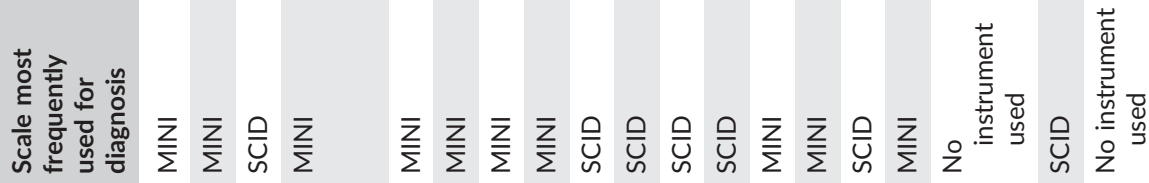

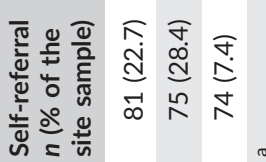

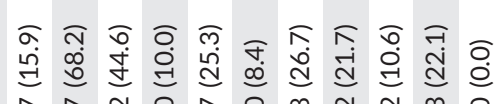

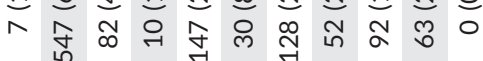

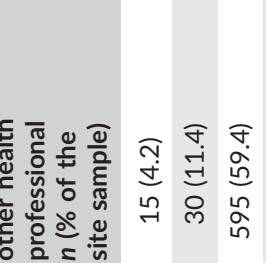

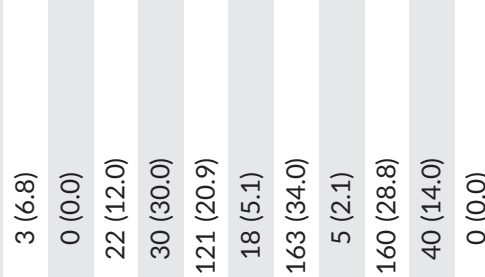

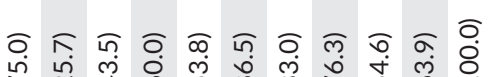

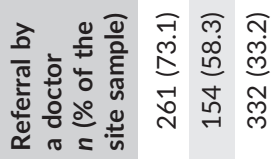

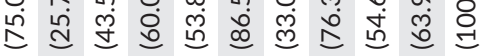

๓)

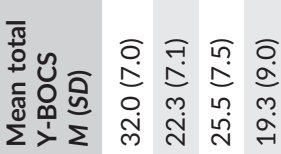

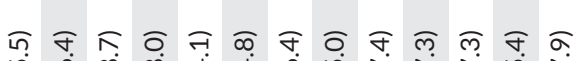

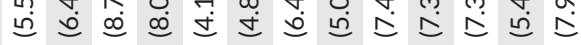

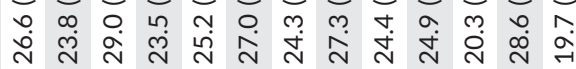

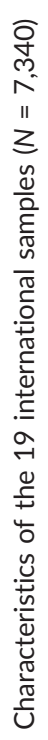

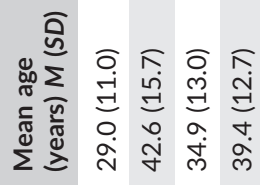

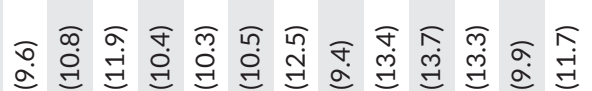

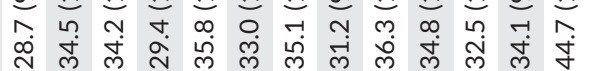

\section{$\frac{\square}{\underline{E}}$}

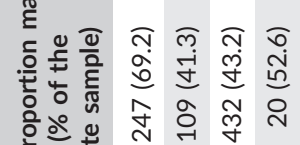

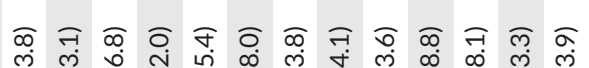

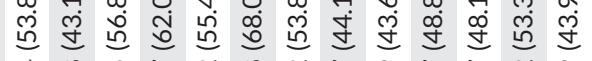

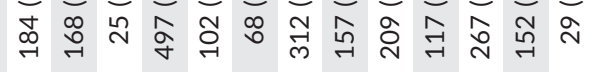

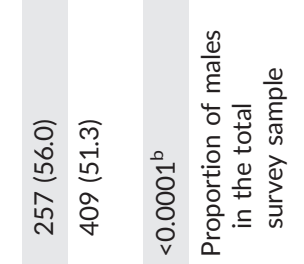

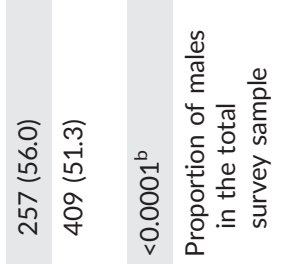

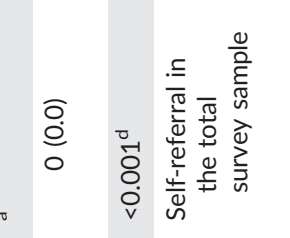

$\frac{T}{\Sigma}$

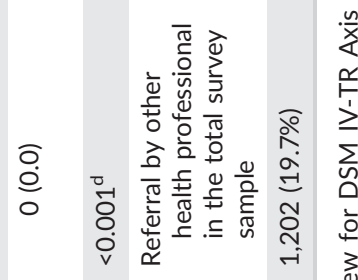

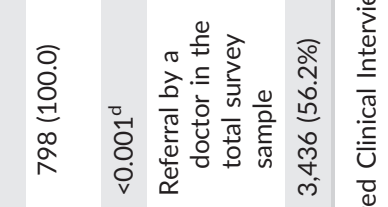

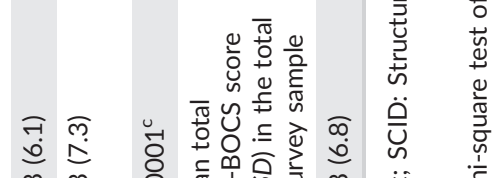

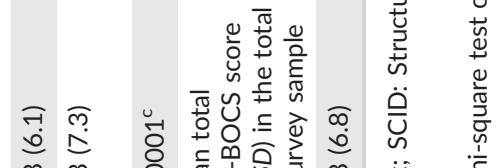

离

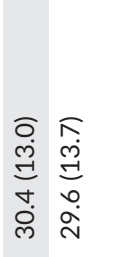

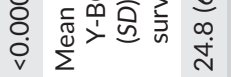

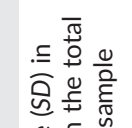

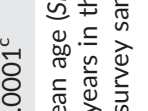

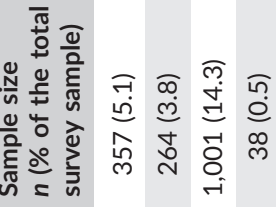

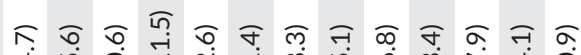

过

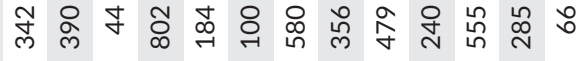

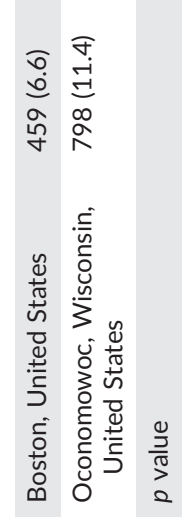

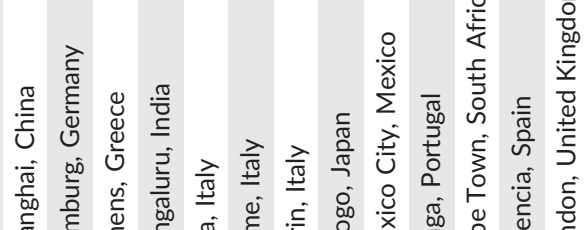

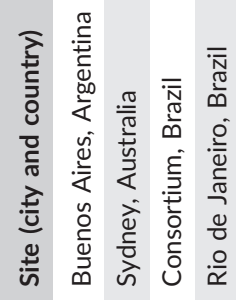

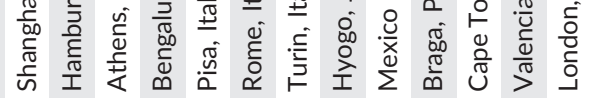

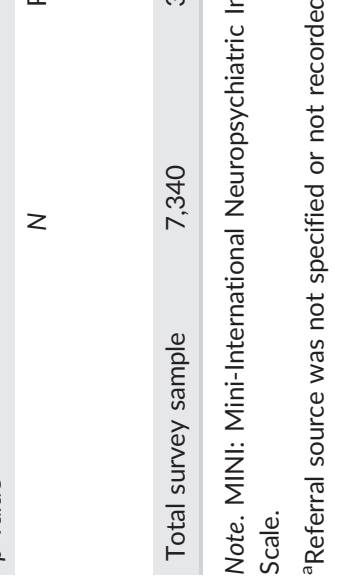


OCD clinics attached to major teaching hospitals that are affiliated with universities. All but two North American and two European centres that were invited participated in the survey. The aim was to collect data on at least one sample from each of the five inhabited continents of the globe. An invitation was also circulated to all members of the International College of Obsessive-Compulsive and Related Disorders (ICOCS). ICOCS is a charitable organisation that aims to further research and public awareness of obsessivecompulsive spectrum disorders, and membership is open to clinicians and academics with interest in the field. Most researchers who participated in the survey are also members of ICOCS. Researchers from 19 specialised OCD centres in 15 countries (Argentina, Australia, Brazil, China, Germany, Greece, India, Italy, Japan, Mexico, Portugal, South Africa, Spain, the United Kingdom, and the United States) responded and completed a standardised data collection sheet using data from their studies. All studies were approved by their respective institutional review boards. The survey assessed medication use crosssectionally, upon referral to each research centre. Information was collected regarding the size of the sample, the years in which the sample was assessed, the mean age and gender distribution of the sample, the mean severity of OCD in the sample, and the mode of referral of participants to the study. Specific enquiry was made regarding the types of psychotropic agents in the following order: SSRIs, serotonin and noradrenalin reuptake inhibitors (SNRIs), clomipramine, other tricyclic antidepressants, mirtazapine, reboxetine, benzodiazepines, atypical antipsychotic medication, typical antipsychotic medication, sodium valproate, lithium, and any other psychotropic medication. There was also specific enquiry in regard to types of psychological therapies and whether any other biological treatments such as TMS, deep brain stimulation, or psychosurgery had been given.
The severity of OCD was assessed with the Yale-Brown Obsessive Compulsive Scale (Y-BOCS; Goodman et al., 1989) in all samples, whereas diagnosis of OCD was made with the Structured Clinical Interview for DSM-IV-TR Axis I Disorders (First, Spitzer, Gibbon, \& Williams, 2007), the Mini-International Neuropsychiatric Interview (Sheehan et al., 1998; Sheehan \& Lecrubier, 2010), or the Anxiety Disorders Interview Schedule for DSM-IV Lifetime Version (Brown, Barlow, \& Di Nardo, 1994).

Consistency of summary statistics across countries was assessed using conventional chi-square tests for categorical variables (i.e., gender, referral type, and medication usage) and the $Q$ heterogeneity statistic for continuous variables (i.e., age and Y-BOCS score). Analyses were performed in SAS version 9.4 (SAS Institute).

\section{3 | RESULTS}

Data were collected on 7,340 participants with OCD from 15 countries. The characteristics of the samples from 19 OCD centres are shown in Table 1. Data were collected between 1990 and 2018. Samples varied significantly on demographic characteristics, for example, gender (the sample from Argentina had more men, and the sample from Australia had more women; overall $p$ value for consistency in gender distribution across counties was $<0.0001$ ) and age (the sample from the United Kingdom was on average 16 years older than the sample from China; overall $p$ value for consistency in age distribution across countries was $<0.0001)$. OCD severity also differed significantly across sites, with the mean Y-BOCS score of the Argentinian sample (32.0, extreme range) being 13 points higher than that of the Rio de Janeiro Brazilian sample (19.3, moderate range; overall $p$ value

TABLE 2 Types of selective serotonin reuptake inhibitors (SSRIs) used in different countries at the time of assessment

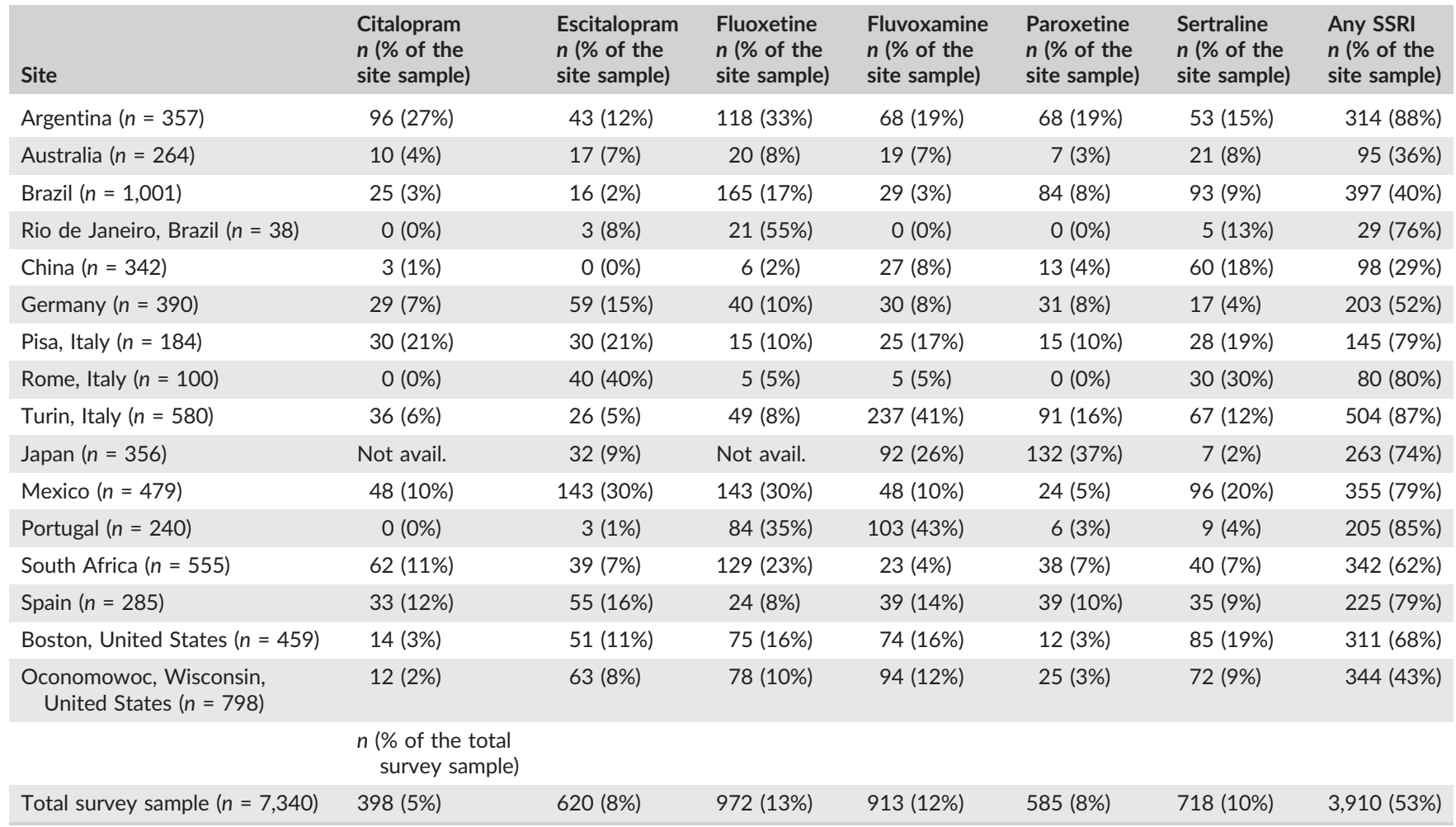


for consistency in Y-BOCS distribution across countries was <0.0001). All participants in the samples from the U.K. and the Boston U.S. centres were referred by a doctor, whereas the majority of the participants from the sample from India ( $n=547 ; 68.2 \%$ ) were self-referred (overall $p$ value for consistency in referral patterns across countries was <0.0001).

Just over one half ( $n=3910 ; 53.3 \%$ ) of the total sample were taking one of the SSRIs (Table 2). There was a significant variation in the rates of SSRI use between different countries (Figure 1), ranging from the lowest in the Chinese sample $(n=98 ; 28.7 \%)$ to the highest in the Argentinian sample ( $n=314 ; 88.0 \%$ ); Table 2 also shows the frequency with which various SSRIs were used in different countries. Citalopram and fluoxetine were not available in Japan, but there was access to all six SSRIs in all other countries. Fluoxetine $(n=972$; $13.2 \%$ ) and fluvoxamine ( $n=913 ; 12.4 \%$ ) were the most commonly used SSRIs, whereas citalopram ( $n=398 ; 5.4 \%$ ) was the least commonly used. Escitalopram was the most frequently used SSRI in the samples from Germany, Rome, and Spain. Paroxetine was the most frequently used SSRI in the Japanese sample. Sertraline was the most frequently prescribed agent in the Chinese sample.

Table 3 shows the frequency of use of other antidepressants and benzodiazepines. Of the SNRIs, only venlafaxine was available in most countries, and it was used in between $2 \%$ and $10 \%$ of the samples. The overall clomipramine use was common ( $n=703$; 11\%), but with a large variability, ranging from $0.3 \%(n=1)$ in the Chinese sample to $39.2 \%(n=140)$ in the Argentinian sample. In the total sample, benzodiazepines were commonly used ( $n=986 ; 15.2 \%$ ), particularly in the Americas, Rome, Japan, and Spain.

Table 4 shows that atypical antipsychotics were used in $23.3 \%$ ( $n=1,368$ ) of the sample. Use of atypical antipsychotics was highest in Argentina ( $n=156 ; 43.7 \%)$ and Japan $(n=154 ; 43.3 \%)$ and lowest in China ( $n=16 ; 4.7 \%)$. Risperidone ( $n=428 ; 7.3 \%)$ and aripiprazole ( $n=415 ; 7.1 \%$ ) were the most commonly used antipsychotics internationally. Less than $5 \%(n=275)$ of the total sample were taking typical antipsychotic agents, with rates being highest in the Spanish and Mexican samples.

Data on the use of mood stabilisers, anticonvulsants, and other pharmacological agents are presented in Table 5. Lithium was the most frequently used mood stabiliser ( $n=238 ; 3.9 \%$ ), and its use was the highest in Italy ( $n=116 ; 13.4 \%)$. Other mood stabilisers and anticonvulsants (i.e., sodium valproate, lamotrigine, carbamazepine, and topiramate) were used less often. Even lower frequencies were reported for psychostimulants (methylphenidate and dexamphetamine), memantine, inositol, $\mathrm{N}$-acetylcysteine, and St. John's wort.

The use of nonpharmacological biological therapies was uncommon. TMS was only used in $23(0.3 \%)$ participants with these coming from Australia, Italy, Japan, Mexico, and the United States. Deep brain stimulation was reported in 11 (0.2\%) participants from Mexico and the United States. Gamma knife surgery was used in five (0.1\%) participants from the Boston site. Psychosurgery involving a craniotomy was reported in $13(0.2 \%)$ participants from Australia, Mexico, and Spain. There were no reports of the use of transcranial direct current stimulation in any of the samples.

Therapy with ERP was received by $31.5 \%(n=1286)$ of the 4,086 participants for whom this information had been recorded. Most centres reported that ERP was usually available in teaching hospitals or specialised clinics and that access to adequately trained ERP therapists was generally difficult, except for Spain and the United Kingdom. Spain reported the highest number of participants who received ERP in the previous/preceding 6 months $(78.9 \%$; $n=225)$. ERP was reported as subsidised by the government in Australia, Germany, Spain, and the United Kingdom. There did not appear to be any relationship between the frequency of ERP and the frequency of SSRI use at each site. The most commonly used psychological therapy after ERP was cognitive therapy, except for Mexico and South America, where psychodynamic therapy was used relatively frequently.

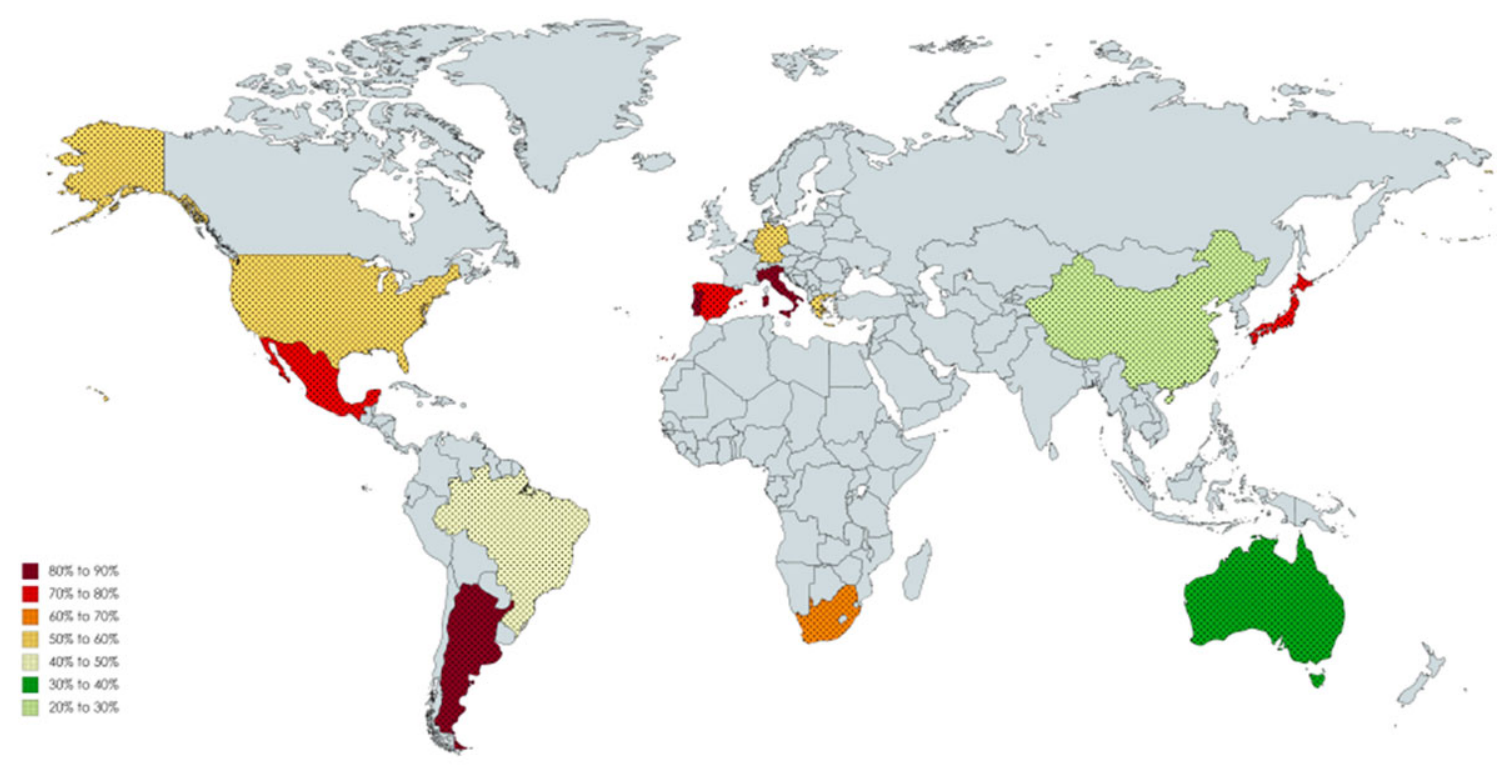

FIGURE 1 World map and the prevalence of selective serotonin reuptake inhibitor use in the countries surveyed 
TABLE 3 The frequency of use of non-SSRI antidepressants and benzodiazepines for obsessive-compulsive disorder among different countries

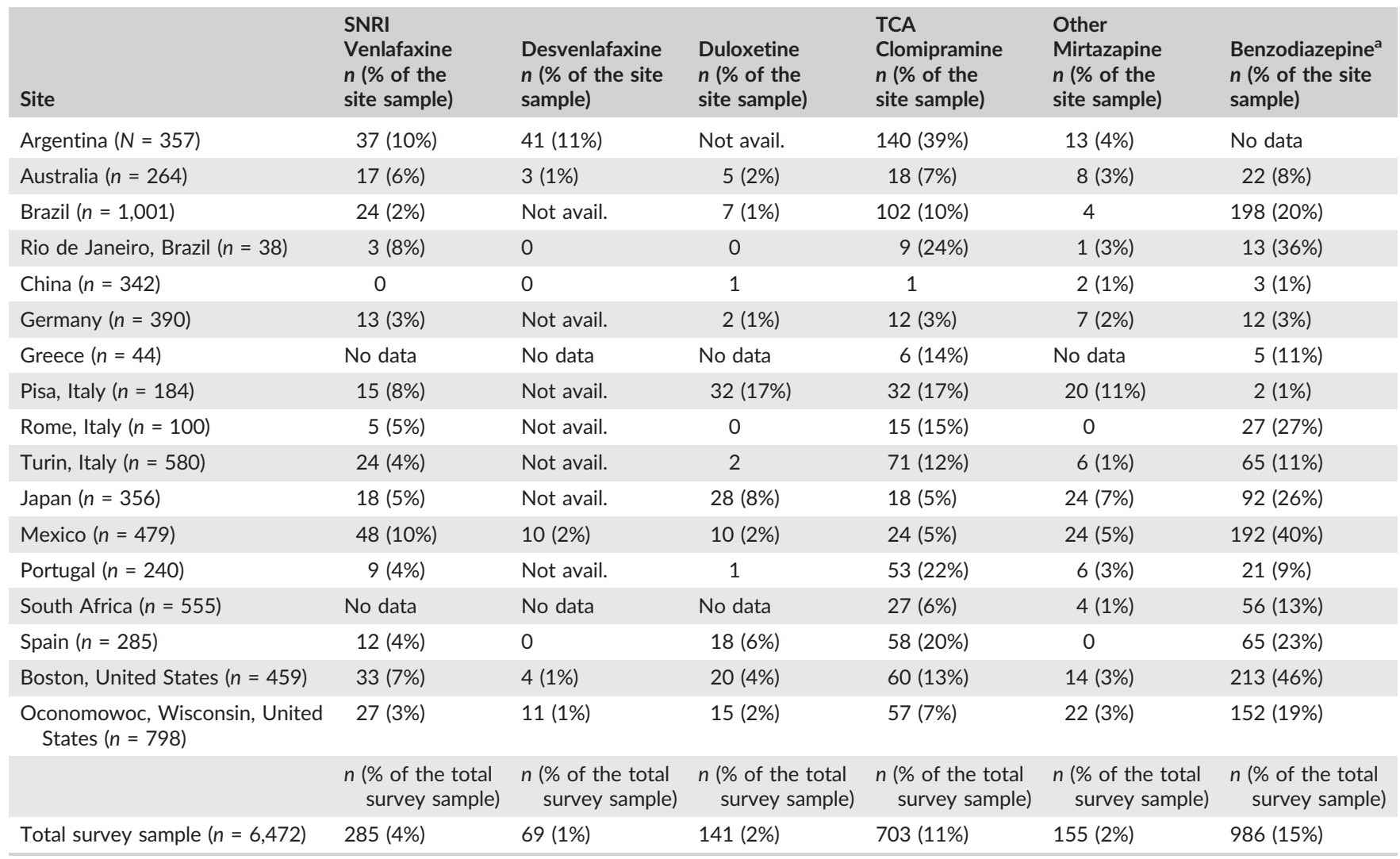

Note. SNRI: Serotonin and noradrenaline reuptake inhibitor; TCA: tricyclic antidepressant.

${ }^{a}$ It was not specified whether benzodiazepines were prescribed regularly, prn, or both regular and prn.

\section{4 | DISCUSSION}

This survey of treatments used in 7,340 participants represents the largest evaluation of prescribing practices for OCD in the literature thus far. In accordance with OCD treatment guidelines, a majority of participants reported taking SSRIs (53.3\%; $n=3,910)$. Despite fluoxetine being the most frequently used SSRI, there was significant variation between sites and this is consistent with previous studies (Brakoulias et al., 2016; Van Ameringen et al., 2014). It is difficult to explain why sertraline was more commonly used in China or why paroxetine was more commonly used in Japan. Factors that may influence the choice of SSRI may include a clinician's experience with the use of a particular SSRI, local marketing practices, varying costs, and availability. Fluoxetine may have been more frequently used as it has been available for the longest period of time. It may also be preferred because of its long half-life and reduced risk of severe discontinuation syndrome. This is clinically relevant, considering a frequent need to switch from one SSRI to another whilst treating OCD. Although different SSRIs can have different treatment effects in different patients, existing research has consistently found that no SSRI is superior to another with regard to treatment outcome for OCD (Skapinakis et al., 2016). Therefore, factors such as side effects of the specific SSRIs, their half-life, availability, cost, and clinician preference may play a role in the choice of SSRIs for OCD.

Clomipramine still has an important role in the treatment armamentarium for OCD, despite its high rate of adverse effects. It was used in $11 \%$ of the entire sample with rates being higher in sites with greater mean OCD severity. For instance, the sample from Argentina had the highest mean $\mathrm{Y}$-BOCS score of all sites and also the highest rate of clomipramine use (39.2\%).

Atypical antipsychotics were used commonly (23.3\%), which is likely to reflect the resistant nature of OCD patients who may have seen several clinicians before coming to a specialised OCD clinic. This finding is similar to the results of a multisite study (Van Ameringen et al., 2014), reporting that $30 \%$ of patients with OCD received augmentation with atypical antipsychotics. The preference for risperidone and aripiprazole as augmenting agents may relate to their relatively favourable side effect profiles and the greater number of reported trials using these agents (Albert et al., 2016; Veale et al., 2014). Most of the sites that were surveyed in this study were collecting data for more than a decade, which might have resulted in an overrepresentation of antipsychotic medication that were introduced earlier, for example, risperidone and olanzapine, compared with antipsychotic medication that appeared more recently, such as paliperidone and lurasidone.

Among other pharmacological agents, this study repeated the findings of the previous survey (Brakoulias et al., 2016) in regard to benzodiazepines. The use of benzodiazepines in approximately $15 \%$ of patients suggests that benzodiazepines have a therapeutic role for some patients with OCD. This role may be related to the treatment of co-occurring anxiety disorders and/or anxiety resulting from distressing ego-dystonic obsessions. The survey revealed low rates 


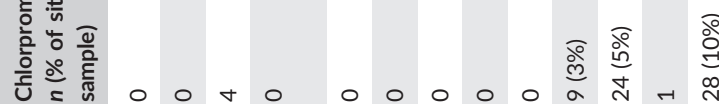

巳

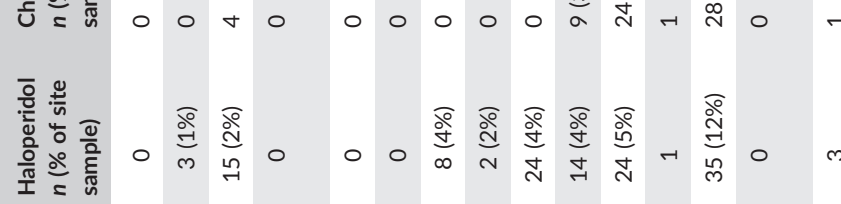

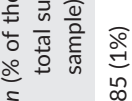

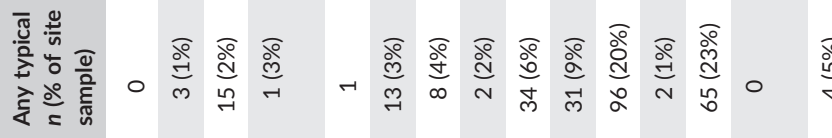

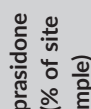

产

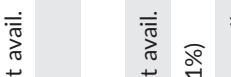

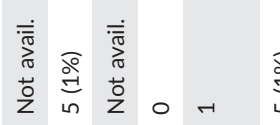

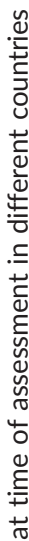

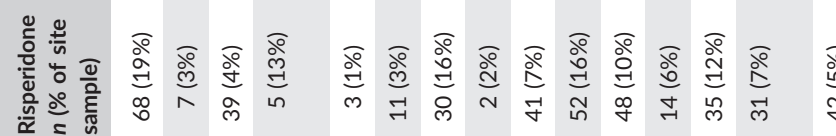

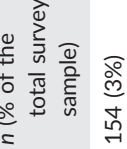

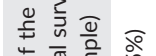

๖ำ

高

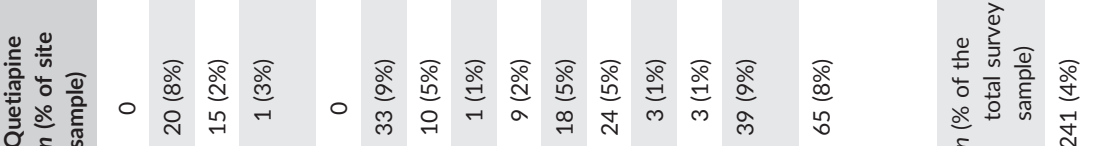

奠 蓄

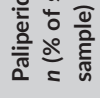

ㅇํㄹ

ঙำ

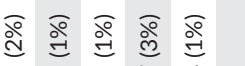

竞竞

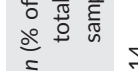

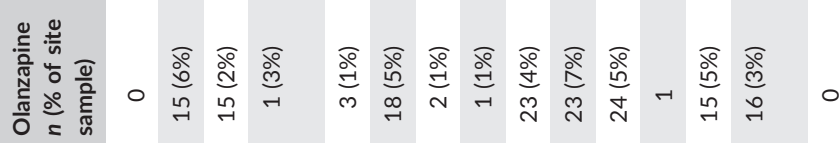

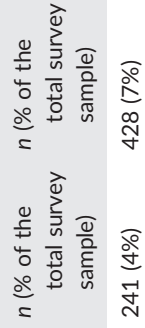

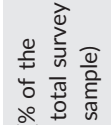

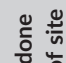

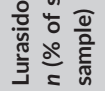

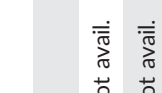

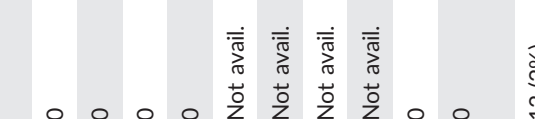

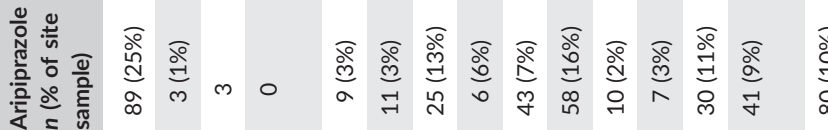

흘

\section{产}

$x_{0}$

○

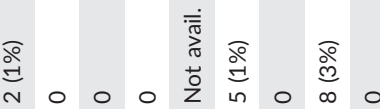

○.

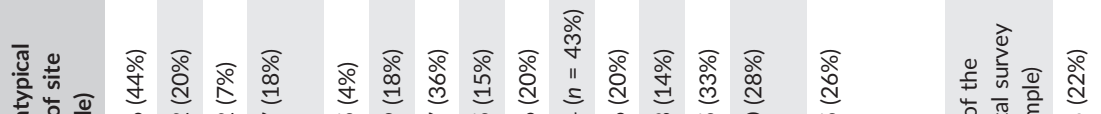

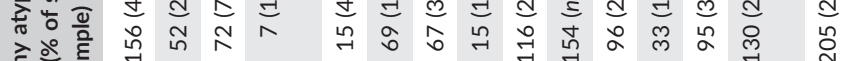

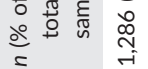

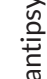

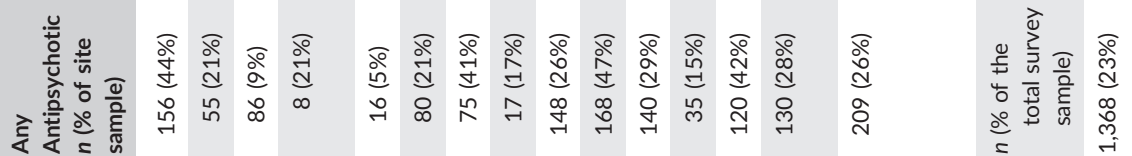
$\stackrel{\circ}{\circ}$ 


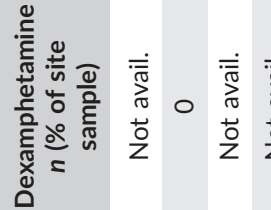

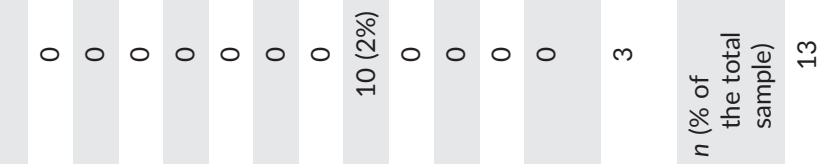

苋

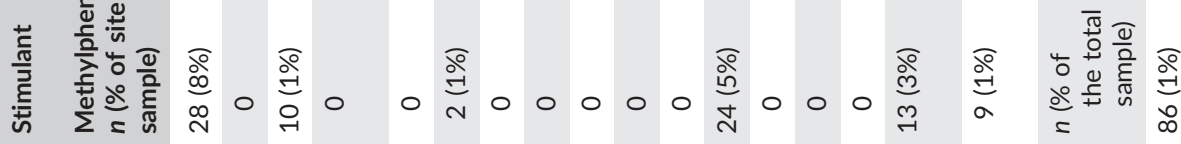

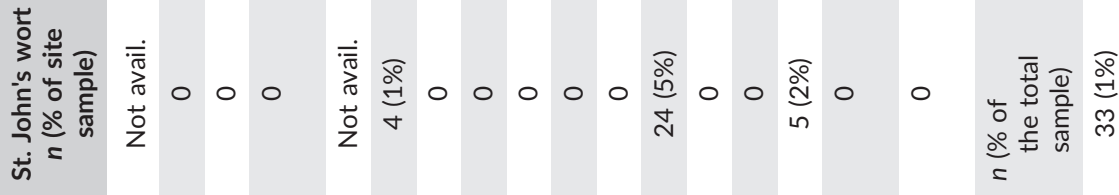

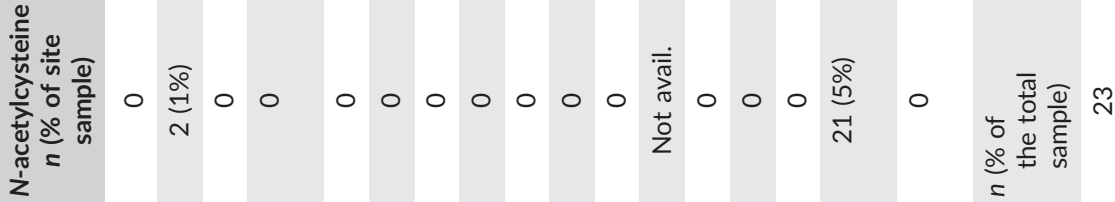

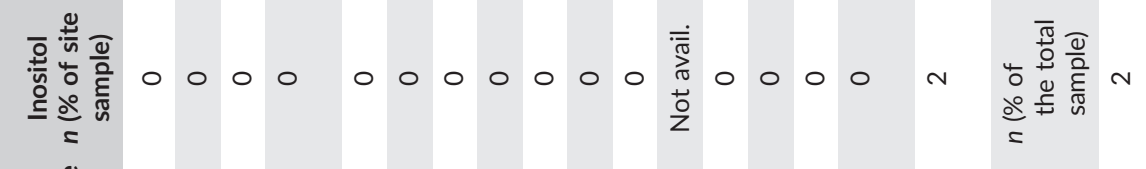

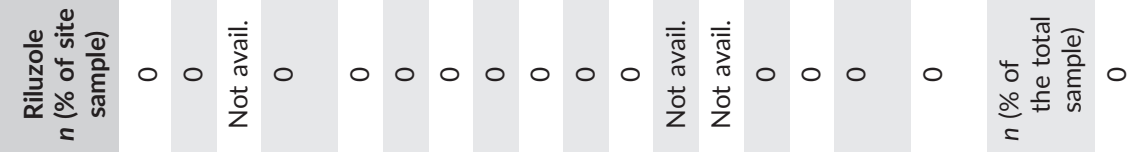

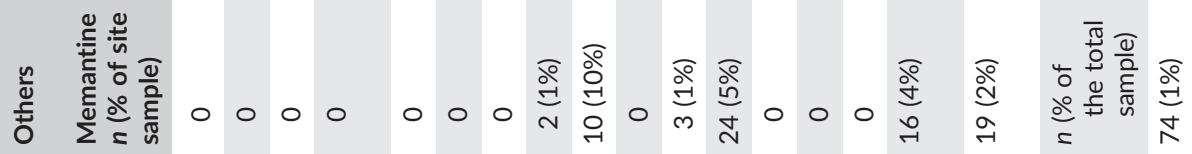

苋

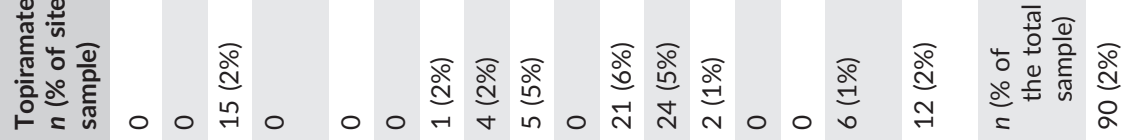

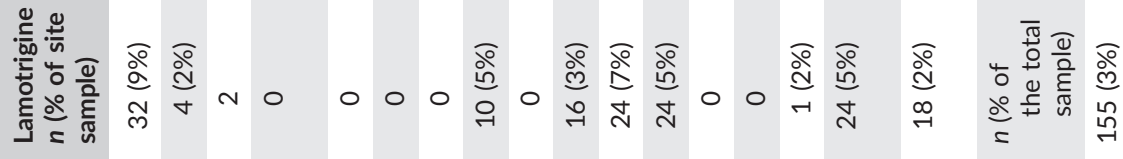

产

㐫

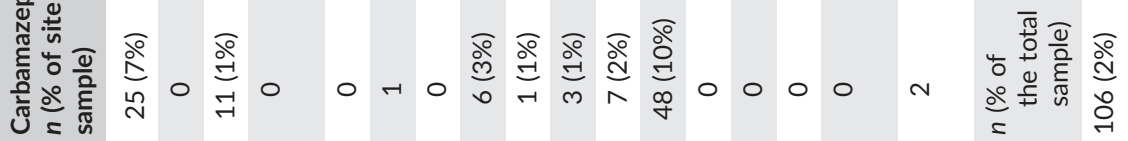

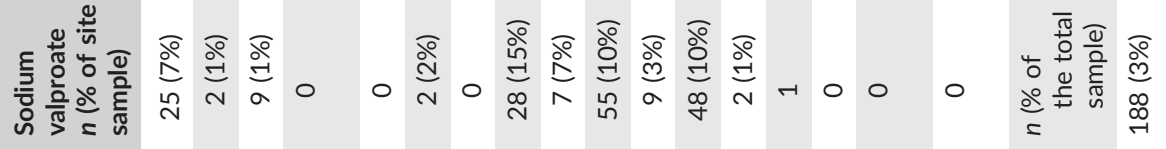

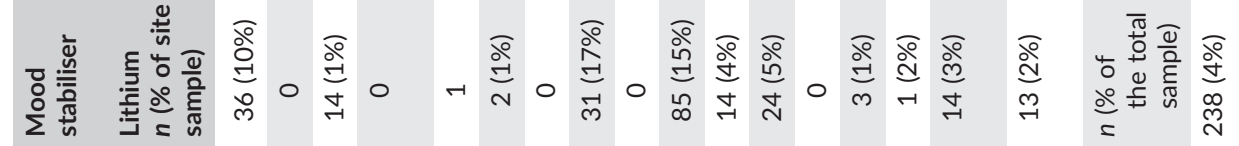

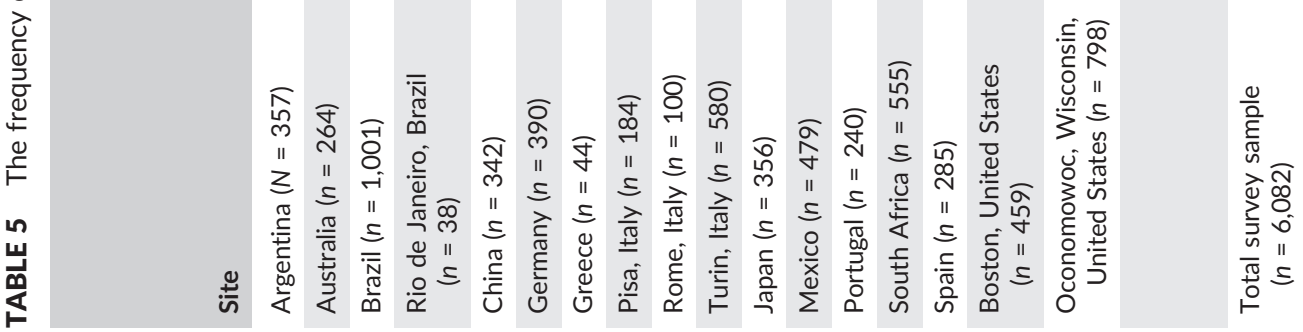


of use of agents thought to act on the glutamate system such as lamotrigine, topiramate, and memantine. Several positive results of trials using glutamatergic agents in OCD have been published (KariukiNyuthe, Gomez-Mancilla, \& Stein, 2014), but these agents are absent from current guidelines, and their role in the treatment of OCD is yet to be ascertained.

The use of TMS has also developed since OCD treatment guidelines were last written, and new treatment guidelines will need to reconsider the role of TMS in the treatment of OCD (Lusicic, Schruers, Pallanti, \& Castle, 2018; Rehn, Eslick, \& Brakoulias, 2018). Defining the role of newer treatment strategies in guidelines encourages clinicians to consider such treatment approaches.

It was surprising that less than a third (31.5\%) of the participants had received ERP in the previous/preceding 6 months, considering that the average Y-BOCS score was in the severe range (24.8) and that the centres were specialised in treating OCD. This relatively low rate of ERP is not in keeping with treatment guidelines and research evidence supporting its use prior to antipsychotic augmentation for OCD (Foa et al., 2007; Simpson et al., 2013). A possible reason for this is a difficulty accessing ERP, so that ERP is "bypassed" and medication is administered instead. It should also be noted that not all centres collected information relating to psychological therapies (the total sample was only 4,086). The Spanish site is led by psychologists, and this is likely to explain the high rate of ERP at this site. There are also studies indicating that ERP is less cost effective when compared with SSRI therapy (Skapinakis et al., 2016).

The rates of SSRI use and ERP within the samples indicate that some patients who were referred to these expert centres were not receiving first-line evidence-based treatments for OCD. Thus, there is benefit in providing education on the treatment of OCD to primary care clinicians. It does appear that centres with samples that reported greater severity as measured by the Y-BOCS (see Table 1) also reported higher rates of SSRI use (see Table 2).

This survey is primarily limited by the heterogeneity within the international sample. Each specialised centre had varying data collection periods, methods of collecting the data, referral criteria, modes of referral, and levels of OCD severity. Some centres did not collect data on certain variables, and this resulted in lower total sample sizes for some variables. It should be emphasised that the low rates of psychotropic use, for example, in China, do not reflect the prescribing practices of the expert centres, but rather the treatments used by participants when presenting to the expert centre for the first time. Although the tables do not suggest the use of multiple antidepressants or antipsychotic agents, the survey did not specifically ask how often multiple pharmacological agents were used. The samples from which the survey results derived were collected over long periods of time, with some centres having collected data since 1990 (the years of data collection at each site have been detailed in Table 1). As discussed, this limits our interpretation of the survey results in regard to the prevalence of antipsychotic augmentation.

The health care systems in regard to access to services and funding models are different in each of the countries. Any future attempt to assess for treatment preferences of referrers to specialist OCD centres should attempt to restrict the dates of data collection, standardise referral criteria, and standardise methods of data collection. The survey also sparks interest in better understanding which treatment strategies are preferred in clinical practice. Future large naturalistic studies may aid our understanding of the real-life treatment response rates, discontinuation rates, and most relevant adverse effects.

\section{5 | CONCLUSION}

In conclusion, this survey provides readers with more detailed international prescribing characteristics in a larger sample than our previous survey. In particular, it shows that fluoxetine and fluvoxamine were among the most frequently prescribed SSRIs and that risperidone and aripiprazole were the most frequently prescribed antipsychotic agents. Novel biological therapies do not appear to be in common use for OCD, and their use may need to be considered in new treatment guidelines. The study also highlighted the underutilisation of ERP and the difficulty that many patients in different countries have in accessing ERP. The findings emphasise the need for updated and well-circulated treatment guidelines for OCD.

\section{ACKNOWLEDGEMENTS}

Author V. B. would like to acknowledge all the staff at the numerous research centres throughout the world and their participants who contributed valuable data to this large data base. V. B. would also like to acknowledge Prof. Naomi Fineberg and the International College of Obsessive-Compulsive Spectrum Disorders (ICOCS) who supported this project by circulating his invitation to participate in the survey to its members.

This study was sponsored by Nepean Medical Research Foundation Grant, Pfizer Neuroscience Grant, National Natural Science Foundation of China (Grant 81371340), and Spanish MINECO Grant PSI2013-44733-R.

\section{CONFLICT OF INTEREST}

The authors have declared no conflict of interest.

\section{ORCID}

Vlasios Brakoulias (10) https://orcid.org/0000-0002-4188-4370

Donatella Marazziti (D) https://orcid.org/0000-0002-4021-5829

\section{REFERENCES}

Albert, U., Carmassi, C., Cosci, F., De Cori, D., Di Nicola, M., Ferrari, S., ... Fiorillo, A. (2016). Role and clinical implications of atypical antipsychotics in anxiety disorders, obsessive-compulsive disorder, traumarelated, and somatic symptom disorders: A systematized review. International Clinical Psychopharmacology, 31, 249-258. https://doi.org/ 10.1097/YIC.0000000000000127

Baldwin, D. S., Anderson, I. M., Nutt, D. J., Allgulander, C., Bandelow, B., Den Boer, J. A., ... Lidbetter, N. (2014). Evidence-based pharmacological treatment of anxiety disorders, post-traumatic stress disorder and obsessive-compulsive disorder: A revision of the 2005 guidelines from the British Association for Psychopharmacology. Journal of Psychopharmacology, 28, 403-439. https://doi.org/10.1177/0269881114525674

Bandelow, B., Sher, L., Bunevicius, R., Hollander, E., Kasper, S., Zohar, J. \& Möller, H. 2012. WFSBP Task Force on Anxiety Disorders OCD and PTSD Guidelines for the pharmacological treatment of anxiety disorders, obsessive-compulsive disorder and posttraumatic stress 
disorder in primary care. International journal of psychiatry in clinical practice, 16, 77-84, https://doi.org/10.3109/13651501.2012.667114.

Brakoulias, V., Starcevic, V., Belloch, A., Dell'osso, L., Ferrão, Y. A., Fontenelle, L. F., ... Matsunaga, H. (2016). International prescribing practices in obsessive-compulsive disorder (OCD). Human Psychopharmacology: Clinical and Experimental, 31, 319-324. https://doi.org/ 10.1002/hup.2541

Brakoulias, V., Starcevic, V., Berle, D., Milicevic, D., Moses, K., Hannan, A., ... Martin, A. (2013). The use of psychotropic agents for the symptoms of obsessive-compulsive disorder. Australasian Psychiatry, 21, 117-121. https://doi.org/10.1177/1039856212470502

Brown, T. A., Barlow, D. H., \& Di Nardo, P. A. (1994). Anxiety Disorders Interview Schedule Adult Version: Client interview schedule. University of Oxford: Oxford University Press.

Eisen, J. L., Sibrava, N. J., Boisseau, C. L., Mancebo, M. C., Stout, R. L., Pinto, A., \& Rasmussen, S. A. (2013). Five-year course of obsessive-compulsive disorder: Predictors of remission and relapse. Journal of Clinical Psychiatry, 74, 233-239. https://doi.org/10.4088/JCP.12m07657

First, M. B., Spitzer, R. L., Gibbon, M. \& Williams, J. B. 2007. SCID-I/P.

Foa, E. B., Liebowitz, M. R., Kozak, M. J., Davies, S., Campeas, R., Franklin, M. E., ... Tu, X. (2007). Randomized, placebo-controlled trial of exposure and ritual prevention, clomipramine, and their combination in the treatment of obsessive-compulsive disorder. Focus, 5, 368-380. https://doi.org/10.1176/foc.5.3.foc368

Goodman, W. K., Price, L. H., Rasmussen, S. A., Mazure, C., Fleischmann, R. L., Hill, C. L., ... Charney, D. S. (1989). The Yale-Brown Obsessive Compulsive Scale. I. Development, use, and reliability. Archives of General Psychiatry, 46, 1006-1011. https://doi.org/10.1001/ archpsyc.1989.01810110048007

Kariuki-Nyuthe, C., Gomez-Mancilla, B., \& Stein, D. J. (2014). Obsessive compulsive disorder and the glutamatergic system. Current Opinion in Psychiatry, 27, 32-37. https://doi.org/10.1097/YCO.0000000000000017

Lusicic, A., Schruers, K. R., Pallanti, S., \& Castle, D. J. (2018). Transcranial magnetic stimulation in the treatment of obsessive-compulsive disorder: Current perspectives. Neuropsychiatric Disease and Treatment, 14, 1721-1736. https://doi.org/10.2147/NDT.S121140

Marazziti, D., \& Consoli, G. (2010). Treatment strategies for obsessivecompulsive disorder. Expert Opinion on Pharmacotherapy, 11, 331-343. https://doi.org/10.1517/14656560903446948

Matsunaga, H., Maebayashi, K., Hayashida, K., Okino, K., Matsui, T., Iketani, T., ... Stein, D. J. (2008). Symptom structure in Japanese patients with obsessive-compulsive disorder. American Journal of Psychiatry, 165, 251-253. https://doi.org/10.1176/appi.ajp.2007.07020340

National Institute for Health and Clinical Excellence. 2005. Obsessive-compulsive disorder: Core interventions in the treatment of obsessivecompulsive disorder and body dysmorphic disorder. [Online]. London National Institute for Health and Clinical Excellence (NICE). Available: www.nice.org.uk/guidelines.aspx?o=guidelines.completed [Accessed 5.1.11 2011].

Rehn, S., Eslick, G. D., \& Brakoulias, V. (2018). A meta-analysis of the effectiveness of different cortical targets used in repetitive transcranial magnetic stimulation (rTMS) for the treatment of obsessivecompulsive disorder (OCD). Psychiatric Quarterly, 89, 1-21.

Ruscio, A., Stein, D., Chiu, W., \& Kessler, R. (2010). The epidemiology of obsessive-compulsive disorder in the National Comorbidity Survey
Replication. Molecular Psychiatry, 15, 53-63. https://doi.org/10.1038/ mp.2008.94

Schruers, K., Koning, K., Luermans, J., Haack, M., \& Griez, E. (2005). Obsessive-compulsive disorder: A critical review of therapeutic perspectives. Acta Psychiatrica Scandinavica, 111, 261-271. https://doi.org/ 10.1111/j.1600-0447.2004.00502.x

Sheehan, D. V. \& Lecrubier, Y. 2010. MINI International Neuropsychiatric Interview English Version 6.0.0 [Online]. Available: http://rucklab. files.wordpress.com/2012/03/mini-6-0-oct-10-2010.pdf [Accessed 23.01.13 2013].

Sheehan, D. V., Lecrubier, Y., Sheehan, K. H., Amorim, P., Janavs, J., Weiller, E., ... Dunbar, G. C. (1998). The Mini-International Neuropsychiatric Interview (M.I.N.I.): The development and validation of a structured diagnostic psychiatric interview for DSM-IV and ICD-10. Journal of Clinical Psychiatry, 59(Suppl 20, 22-33;quiz), 34-57.

Simpson, H. B., Foa, E. B., Liebowitz, M. R., Huppert, J. D., Cahill, S., Maher, M. J., ... Williams, M. T. (2013). Cognitive-behavioral therapy vs risperidone for augmenting serotonin reuptake inhibitors in obsessivecompulsive disorder: A randomized clinical trial. JAMA Psychiatry, 70, 1190-1199. https://doi.org/10.1001/jamapsychiatry.2013.1932

Skapinakis, P., Caldwell, D. M., Hollingworth, W., Bryden, P., Fineberg, N. A., Salkovskis, P., ... Churchill, R. (2016). Pharmacological and psychotherapeutic interventions for management of obsessive-compulsive disorder in adults: A systematic review and network meta-analysis. The Lancet Psychiatry, 3, 730-739. https://doi.org/10.1016/S22150366(16)30069-4

Skoog, G., \& Skoog, I. (1999). A 40-year follow-up of patients with obsessive-compulsive disorder. Archives of General Psychiatry, 56, 121. https://doi.org/10.1001/archpsyc.56.2.121

Sorsdahl, K., Blanco, C., Rae, D. S., Pincus, H., Narrow, W. E., Suliman, S., \& Stein, D. J. (2013). Treatment of anxiety disorders by psychiatrists from the American Psychiatric Practice Research Network. Revista Brasileira de Psiquiatria, 35, 136-141. https://doi.org/10.1590/1516-44462012-0978

Stein, D. J., \& Rapoport, J. L. (1996). Cross-cultural studies and obsessivecompulsive disorder. CNS Spectrums, 1, 42-46. https://doi.org/ 10.1017/S1092852900000675

Van Ameringen, M., Simpson, W., Patterson, B., Dell'osso, B., Fineberg, N., Hollander, E., ... Karamustafalioglu, O. (2014). Pharmacological treatment strategies in obsessive compulsive disorder: A cross-sectional view in nine international OCD centers. Journal of Psychopharmacology, 28, 596-602.

Veale, D., Miles, S., Smallcombe, N., Ghezai, H., Goldacre, B., \& Hodsoll, J. (2014). Atypical antipsychotic augmentation in SSRI treatment refractory obsessive-compulsive disorder: A systematic review and metaanalysis. BMC Psychiatry, 14, 317. https://doi.org/10.1186/s12888014-0317-5

How to cite this article: Brakoulias V, Starcevic V, Albert U, et al. Treatments used for obsessive-compulsive disorderAn international perspective. Hum Psychopharmacol Clin Exp. 2019;34:e2686. https://doi.org/10.1002/hup.2686 